\title{
The importance of metacognition and the experiential learning process within a cultural intelligence-based approach to cross-cultural coaching
}

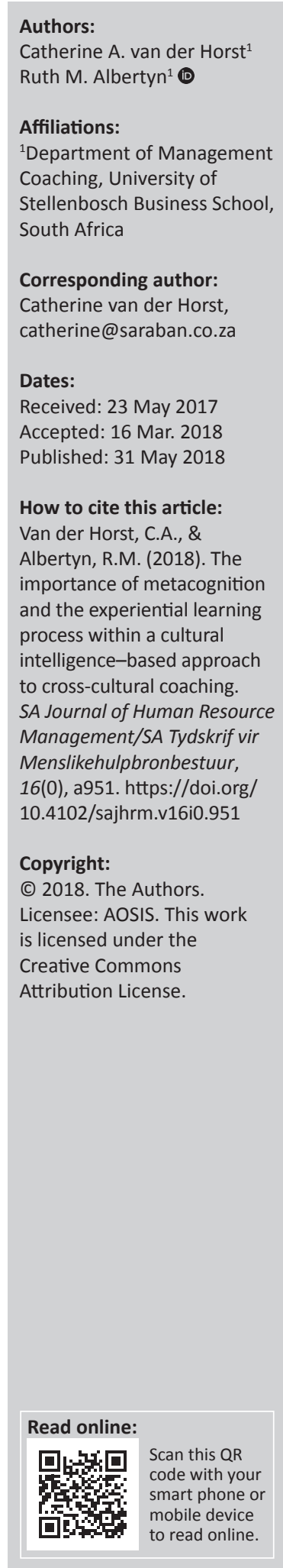

Orientation: Research on cultural intelligence (CQ) is increasingly used to evaluate, explain and predict the cross-cultural efficacy of management behaviour in everyday cross-cultural interactions. However, there is limited evidence in cross-cultural coaching of the use of a CQ-based approach incorporating metacognition and experiential learning theory (ELT).

Research purpose: This article explored the theoretical linkages, benefits and directions of CQ for enhancing cross-cultural coaching.

Motivation for the study: Exploration of theoretical perspectives of CQ for application in cross-cultural coaching.

Research design, approach and method: A critical interpretative synthesis research methodology was employed to identify and study key concepts. The methodology is sensitive to the emergence of meaning in a diverse body of literature from adjacent disciplines.

Main findings: This research suggests four findings motivating a CQ-based approach for cross-cultural coaching: firstly, the recognition of the use of metacognitive strategies in (cross-cultural) coaching; secondly, the usefulness of metacognition to cross-cultural coaching for grasping and transforming cultural experience and insights into culturally appropriate behaviour; thirdly, an understanding of the significance of suitability and predisposition of certain learning styles to cross-cultural learning effectiveness and lastly, acknowledging the importance of a heightened focus on the experiential learning process within the crosscultural coaching engagement.

Practical and managerial implications: Key concepts and insights from research on CQ have application in cross-cultural coaching in pursuit of the transformation of cultural awareness and insight into culturally appropriate behaviour.

Contribution/value-add: This research motivates the use of a CQ-based approach incorporating metacognition and ELT to cross-cultural coaching.

\section{Introduction}

Cross-cultural coaching is an emerging and increasingly important field owing to the acceleration of globalisation and increased labour mobility. Intercultural collaboration has become essential in the everyday workplace, and management behaviour has been recognised as a key driver for navigating and overcoming some of these cross-cultural challenges (Deng \& Gibson, 2008; Dickson, Den Hartog, \& Mitchelson, 2003; Rosinski, 2003). Reflective practice is used extensively in coaching to cultivate insight and facilitate personal development. This article focuses on the metacognitive strategies of awareness, mindfulness and perspective-taking (established forms of reflective practice) that can lead to improved mental agility, contextual thinking and cognitive flexibility necessary to transform knowledge and experience into culturally appropriate behaviour. Furthermore, this article examines the integration of cultural intelligence (CQ) and Kolb's experiential learning theory (ELT), and the contribution that this can potentially make to the efficacy of cross-cultural coaching. Insights from this article could provide cross-cultural coaches with a more-integrated mechanism and tools to facilitate the development of CQ in a multicultural setting.

\section{Purpose}

Failure to understand and respect cross-cultural difference invariably leads to personal and professional frustration, an inability to build trust and achieve consensus, wasted time, missed 
business opportunities and loss of revenue. When the nuances in culturally diverse settings are not fully appreciated, misread or overlooked, it can lead to delays in getting agreement and contracts concluded, sometimes leading to the overall failure of a transaction (Rowland, 2016). Although the importance of addressing these challenges is clear, it remains unclear how this skill can be learnt and adopted.

Cross-cultural coaching is a response to navigating culturally diverse situations. Coaching stands in conjunction with and is complementary to other support services to improve CQ, such as training and mentoring. The literature offers many definitions of cultural or cross-cultural competence that generally include 'the ability to function effectively in another culture' (Johnson, Lenartowicz, \& Apud, 2006; Tan \& Chua, 2003). Rosinski (2003) states that cultural differences should be embraced and not dismissed. Coaching in a crosscultural setting can make a contribution by assisting the client to engender culturally appropriate behaviour through an increased sense of awareness, resulting in greater mental agility in a given situation. Booysen (2015) defined crosscultural coaching as:

\footnotetext{
... a meaning making process, in which the coach helps the coachee to surface and address deeply held beliefs and behaviors, based on cognitive schemas and frameworks shaped by culture and identity constructions, that inhibit their performance in their current context. (p. 242)
}

Plaister-Ten (2009) adds that cross-cultural coaching focuses on awareness of cultural difference and working within these differences to find culturally appropriate steps to address issues. The mechanism whereby experience and knowledge are transformed into culturally appropriate behaviour has not been meaningfully integrated into cross-cultural coaching. The aim of this article is to provide theoretical perspectives on the use of a CQ-based approach to cross-cultural coaching.

It is argued that three insights from the research on CQ are applicable to cross-cultural coaching. Firstly, it is argued that the use of metacognitive strategies through different forms of reflective practice, namely, awareness, perspective-taking and mindfulness, facilitate the transformation of knowledge and experience into culturally appropriate behaviour. Secondly, there are significant interactions and linkages between the components of CQ and the stages of Kolb's experiential learning cycle. Lastly, Kolb's experiential learning styles (ELS) have a moderating impact on the ability to improve CQ. Awareness of the theoretical linkages between metacognition, ELT and cross-cultural coaching provides coaches with insight into ways to facilitate the development of CQ in a multicultural setting.

\section{Literature review}

The construct of CQ is the psychological measure of cultural competence, defined as a person's capability to function effectively in settings characterised by cultural diversity (Earley \& Ang, 2003). This ability to adapt one's behaviour to a new cultural setting, that is, the transformation of inner awareness to appropriate external behaviour, makes CQ unique and is the first step to shaping a cross-cultural interaction. The concept has been developed, tested and used in research on expatriates, leadership, judgement and decisionmaking in culturally diverse settings (Ang et al., 2007; Elenkov \& Manev, 2009). Similar to other forms of intelligence, CQ has both internal (metacognitive, cognitive and motivational) and external (behavioural) components (Earley \& Ang, 2003), and captures awareness and flexibility in intercultural situations (Oolders, Chernyshenko, \& Stark, 2008).

Two nascent strands of research have emerged from recent literature on CQ that in part addresses the improvement of CQ abilities. The first recognises the importance of metacognitive strategies (Ang et al., 2007; Thomas, 2006), namely, awareness, perspective-taking and mindfulness in the process of improving CQ. The literature on CQ and metacognition has begun to establish an empirical basis that demonstrates the positive relationship between metacognition and a number of behavioural measures in culturally diverse settings (Mor, Morris, \& Joh, 2013). The second is concerned with the connection between Kolb's ELT (Kolb, 1984) and $C Q$, which investigates the linkages between Kolb's learning stages and the components of CQ, and the moderating impact of Kolb's learning styles on the improvement of CQ (Van Dyne, Ang, \& Livermore, 2009).

Traditionally, cross-cultural coaching has been preoccupied with questions of cultural profiling (Hampden-Turner \& Trompenaars, 1997; Hofstede, 1981; House, Hanges, Javidan, Dorfman, \& Gupta, 2004; Javidan \& House, 2002), paradigmatic approaches (Geertz, 1973; Hofstede, 1981), culturally bound awareness (Plaister-Ten, 2013; St Claire-Ostwald, 2007) and cultural mapping (Meyer, 2014) amongst a number of research questions. Rosinski (2003, p. xix) states that coaching across cultures is a 'more creative form of coaching'. Furthermore, Plaister-Ten (2013, p. 54) refers to the importance of 'culturally appropriate responsibility' in cross-cultural coaching. Crosscultural coaching has been linked to CQ by Booysen (2015, p. 242) who used terms such as 'cultural agility' and 'cultural humility' during the process of coaching. Plaister-Ten (2013) calls for a systems approach to coaching in intercultural contexts. Notwithstanding the translation of the construct of CQ into accessible coaching practitioners' concepts (Livermore, 2009), the literature on cross-cultural coaching (Abbott, Gilbert, \& Rosinski, 2013; Booysen, 2015; DeLay \& Dalton, 2006; Jenkins, 2006; Rosinski \& Abbott, 2006) has otherwise given limited attention to the thorough examination of the combination of the theory of CQ and the role of metacognitive strategies and ELT in cross-cultural coaching, which will be the focus of this article.

\section{Method \\ Research approach}

Given the decision to carry out a conceptual study, there were three high-level methodological choices necessary in selecting an appropriate method of synthesis, namely, concept analysis, 
interpretative synthesis and systematic review. Each method represents its own school of thought and presents a set of research benefits and challenges to be considered in line with the best fit of methodology to the research question. The methods span a continuum where concept analysis, on the one hand, is founded on qualitative research, and systematic review, on the other hand, is founded on quantitative research (BarnettPage \& Thomas, 2009, p. 59). Interpretative synthesis is posited between these two research methods and demonstrates elements of both qualitative and quantitative research.

The critical interpretative synthesis (CIS) methodology, developed by Dixon-Woods et al., is 'sensitised to the process of conventional systematic review and draws on recent advances in methods for interpretative synthesis' (Dixon-Woods et al., 2006, p. 2). Critical interpretative synthesis supports the analysis of a large, diverse and complex body of literature where definitions were not consistently operationalised, and which generates theory with strong explanatory power (Dixon-Woods et al., 2006). In this research, it was neither the intention to exclusively track the development of concepts nor was it possible to conduct a systematic review, as this requires a basic comparability of data, a priori definition of key concepts and clearly defined inclusion and exclusion criteria (Dixon-Woods et al., 2006). The intention was to examine, through an iterative process, the emergence of meaning of key and supporting concepts scattered across a body of literature from overlapping research disciplines - in this case, studies of intelligence and cognition, psychology, anthropology, and coaching. This method allowed recurring themes to emerge and a critique to be developed. Critique of the literature was undertaken in a dynamic, recursive and reflexive manner and recognised the ill-defined boundaries and overlapping fields presented in this emerging field of study. The analysis developed a synthesizing argument, which was to connect the insights on CQ to cross-cultural coaching and to link key concepts and constructs meaningfully, through newly developed synthetic constructs, in a coherent theoretical framework.

\section{Design}

This CIS design was selected for its flexibility and ability to deal meaningfully with the many exploratory dimensions of the research on cross-cultural coaching and CQ from the definitional issues to the extraction of the prominent themes within overlapping disciplines. Coaching borrows heavily, draws widely and integrates research from fields as diverse as psychology, neuroscience, anthropology and the management sciences, amongst others. Its focus is foremost on practical applications, yet there is limited empirical research available to support key ideas and practices in coaching. There is therefore little consistency in the research approach, unit of measurement or adoption of research paradigm in addressing research questions. This challenge raised questions of credibility and trustworthiness of the primary data of this research assignment. To ensure consistency and rigour, it was necessary to address the credibility of the literature related to the field of coaching.

\section{Targeted body of literature}

The selection of relevant literature was the result of the theoretical needs of the research and the choices best suited to the research purpose (Schreiber, Crooks, \& Stern, 1997, p. 319). This research sought to further the conceptual ordering and clarification of the field of study. In this regard, the development of mid-range theory was an important outcome.

The literature search and selection process evaluated a body of literature significantly larger than the listed references included in the final publication of the research. ${ }^{1}$ Although, a large amount of literature was not overtly relevant to the subject, it nevertheless assisted in informing and understanding the theoretical context in which CQ and key related research efforts developed.

\section{Findings}

Clarity regarding the construct of CQ precedes the discussion of the metacognitive strategies which could be used in crosscultural coaching.

\section{The theoretical construct of cultural intelligence}

As this article proposes a CQ-based approach for cross-cultural coaching, it is important to provide a cursory introduction to the concept of CQ. Despite a proliferation of CQ-related research and other models of CQ (Earley \& Mosakowski, 2004; Thomas \& Inkson, 2003), the model of CQ introduced by Earley and Ang (2003) remains at the centre of research efforts. Cultural intelligence seeks to explain the dimensions of intelligence that facilitates the grasping and transforming of experience in order to create the capacity for effectiveness in cross-cultural situations. A defining feature of CQ is that it is an etic construct. It is universal, independent of culture and is an intelligence capability present in all persons. By contrast, an emic construct is ethnocentric. Its meaning is derived from the context and is culturally bound (Earley, Murnieks, \& Masakowski, 2007). The etic character of the construct of CQ is an important attribute as the insights from CQ have application in cross-cultural coaching, regardless of the cultural context. The theory and conceptual model of CQ comprises four components: metacognition, cognition, motivation and behaviour, explained below in more detail.

Metacognition, an innate and universal attribute, is concerned with the structure and form of thought, that is, 'thinking about thinking' (Earley \& Gibson, 2002, p. 100). It is the process that individuals use to acquire and understand knowledge, in other words, categories of thought, levels of analysis and strategies for information acquisition. It transcends culture and is not concerned with the content of

1.As an example, a Google Scholar search reports almost 1000 articles that contain the term cultural intelligence in the title. A search of articles referencing Robert the term cultural intelligence in the the $A$ search of articles ref Sternberg, the father of modern-day studies on intelligence, and the exact term cultural intelligence, returns 920 articles. Clearly, this is an impossible task. The search strategy thus required further narrowing of the search with a number of qualifying terms, for example, metacognition or coaching. Once articles, possibly of interest and significance, were identified, pearl stringing was used to trace key piece of literature. This greatly assisted in understanding the emergence of key themes and academic focus. 
one culture or another, but rather with the mental processes of representation (Sternberg, 2004, p. 328). Metacognition provides the key capability for the transformation of cognition into directed culturally appropriate behaviour.

Cognition is concerned with the elements that constitute a specific cultural context and account for differences in behaviour and interactions across different cultural settings (Ang \& Van Dyne, 2008). It includes the knowledge structures of 'norms, practices, and conventions in different cultures acquired from education and personal experiences. This includes knowledge of economic, legal, sociolinguistic, and interpersonal systems of different cultures and subcultures' (Ng, Van Dyne, \& Ang, 2012, p. 33). Cognition is the content of experience.

The motivational component of CQ is the energy and interest directed at situations marked by cultural difference (Ang \& Van Dyne, 2008). Motivation plays a central role in the alignment of metacognition, cognition and behaviour, and provides the impetus for culturally appropriate behaviour.

Behaviour as a component of CQ is the ability to enact appropriate verbal and non-verbal actions when interacting with people from different cultures (Ang \& Van Dyne, 2008). As an outward manifestation or overt action, that is, what a person does or says rather than what he or she thinks, behaviour is the basis upon which perception is formed by others in cross-cultural settings.

The theory of CQ has been extensively tested and validated (Ng et al., 2012, pp. 30-31). As a theory with particular emphasis on metacognition, it provides numerous insights that can support the transformation of experience into culturally appropriate behaviour. Applied to the domain of coaching, CQ provides a robust theoretical and practical foundation for cross-cultural coaching. The literature on $C Q$ identifies and continues to confirm the empirical validity of metacognition as the key component responsible for the transformation of experience and insight into culturally appropriate behaviour (Ang \& Van Dyne, 2008; Chua, Morris, \& Mor, 2012; Mor et al., 2013; Thomas \& Inkson, 2003; Van Dyne et al., 2012). It comprises the processes of monitoring and adjusting one's thoughts, planning and strategising as one learns new skills (Schoenfeld, 1987; Silver, 1987; Thomas \& Inkson, 2003; Triandis, 1995). Metacognition is identified as the key enabling faculty in the construct of CQ as it links the cognitive and behavioural elements of CQ by facilitating the translation of thought into action owing to ongoing active awareness that leads to situation-appropriate behaviour. Metacognition, the process involved in developing a heightened sense of CQ (Li \& Mobley, 2010; Li, Mobley, \& Kelly, 2013), is central to the process of cross-cultural coaching and can assist with the improving of CQ.

Recent literature on CQ highlights the importance of metacognition and the use of various metacognitive strategies to enhance CQ (Chua et al., 2012; Mor et al., 2013; Van Dyne et al., 2012). References in the literature to the metacognitive strategies of awareness, perspective-taking and mindfulness provide numerous touch points for cross-cultural coaching. These metacognitive strategies form an integral part of the learning process (Kolb, 1984). Despite limited definitional clarity and a large degree of overlap, cross-cultural coaching can facilitate the individual development of metacognitive strategies by seeking to improve awareness, engaging in perspective-taking and cultivating mindfulness, leading to improved mental agility, increased agency, contextual thinking and cognitive flexibility. An understanding of the importance of developing metacognition and the usefulness of metacognitive strategies is empowering to both coach and client in the cross-cultural coaching context.

\section{Metacognitive strategies}

Metacognitive strategies provide coaching tools that allow the coach to better understand the basis of engagement with the client and areas for development. For the client, the reflective process creates an awareness of potential blind spots and enabling tools for improved cross-cultural interaction.

\section{Awareness}

Awareness, the first metacognitive strategy relevant to $C Q$ and cross-cultural coaching, can be defined as the 'continuous monitoring of one's internal state and the external environment' (Thomas, 2006, p. 94). It is a sense-making activity comprising self-awareness, other-awareness and situational-awareness (Endsley, 1995; Sheldon, 1996; Triandis, 2006). The literature on coaching is replete with the importance and significance of awareness. Booysen (2015) asserts that awareness of cultural understandings can help with interpreting what takes place in diverse contexts. Rosinski (2003) notes that cultural awareness goes beyond a recognition of cultural difference, and that this awareness is in fact concerned with learning about, appreciating and recognising the invisible behavioural influence in everyday interactions and valuing cultures different from one's own. Whitmore describes awareness as 'high-quality self-generated relevant input', that is, being conscious of what is going on around one and demonstrating the self-awareness to be conscious of what one is experiencing (Whitmore, 2009, pp. 33-36).

\section{Perspective-taking}

Multiple perspectives, a form of reflective practice, can also be understood as perspective-taking. Cultural perspectivetaking can be defined as 'considering the typical approach that a counterpart from another culture might take' (Lee, Adair, \& Seo, 2013, p. 390). A number of empirical studies have highlighted the importance and significance of cultural perspective-taking. Mor et al. (2013) found empirical support for the idea that cultural perspective-taking can improve metacognition and intercultural effectiveness, and in turn, CQ. Research by Galinsky (2002) and Galinsky and Moskowitz (2000) found that cultural perspective-taking can reduce confirmation bias and stereotyping. The literature emphasises that contextual thinking and cognitive flexibility, two important aspects of metacognition, underpin cultural 
perspective-taking (Klafehn, Banerjee, \& Chiu, 2008; Lee et al., 2013). The former aspect is concerned with a heightened sensitivity to and awareness of the fact that culture shapes motivation and behaviour, whereas the latter denotes the selective basis upon which mental schema and behavioural scripts are used in cross-cultural situations. The literature suggests that cultural perspective-taking is an ongoing activity for persons with higher levels of metacognition (Klafehn et al., 2008). Booysen (2015, p. 273) refers to reflective and reflexive practice as being vital when developing metacognition.

\section{Mindfulness}

According to the literature in the management sciences, a meaningful and positive connection exists between mindfulness and appropriate behavioural outcomes. These findings include the impact of mindfulness on positive regard, influence and relational and instrumental outcomes (Kopelman, Avi-Yonah, \& Varghese, 2011), and enhanced task performance (Dane, 2011). The concept of mindfulness is widely used, but with varying definitional clarity. The modern-day concept emanates from the literature on psychology (Langer, 1989) and is defined as 'the awareness that emerges through paying attention on purpose, in the present moment, and nonjudgmentally to the unfolding experience moment by moment' (Kabat-Zinn, 2003, p. 145), that is, directing heightened awareness and enhanced attention to the present reality (Brown \& Ryan, 2003). Mindfulness, a conscious discipline (Walsh, 1980), and a product of reflective practice, controls the cognitive processes that govern behaviour by recalling knowledge that is pertinent to the situation, preventing automatic responses, suspending inappropriate responses and constructing responses that are situation-appropriate and consistent with the motivations of the individual (Logan, 1989). The metacognitive strategy of mindfulness focuses attention on the knowledge of culture and process of influence as it pertains to culture by creating new mental maps through the ongoing categorisation and re-categorisation of experience. Mindfulness draws attention to the motives, goals, emotions and external stimuli of the individual, enables the consideration of appropriate behavioural alternatives and creates the capacity for empathy and culturally sensitive and appropriate responses (Thomas, 2006, pp. 85-86).

\section{Discussion}

Coaching tools, derived from metacognition, ELT and CQ, are applied to the cross-cultural context.

\section{Cross-cultural coaching applications of metacognition}

Awareness, perspective-taking and mindfulness, as metacognitive strategies, work hand in hand and overlap in terms of theory and reflective coaching technique.

Building awareness forms the starting point of the crosscultural coaching engagement (Whitmore, 2009). The coach can draw on a number of coaching techniques to challenge assumptions, recognise prejudice, acknowledge blind spots, explore stereotypes and engage in Socratic questioning, to mention a few. It is important to recognise that the coach also brings assumptions and prejudices to the coaching engagement. As part of reflective practice, building awareness is an ongoing focus of the cross-cultural coaching engagement.

In the case of perspective-taking, transforming the experience is an essential part of building a recognition and acceptance of the plurality of perspectives. The coach works with the client to explore, challenge and learn from direct observations and personal experiences. This is a two-step process of understanding experience and then encouraging the client to engage in experiences based on this new understanding. In this way, the learning enables the client to adapt to new ways of thinking about and engaging with their environment. It is worth noting that the coach, similarly, brings his or her perspectives to bear on the coaching engagement and that this may have an impact on the way in which the coach is able or unable to support the client.

Mindfulness, in cross-cultural coaching, draws together awareness and perspective-taking, by focusing attention on automatic thoughts and their effects, exploring instances where the client could have made a different or culturally appropriate choice and cultivating more deliberate and purposeful thought directed at the present. The cross-cultural coaching process facilitates the engagement of thoughts and emotions of the client with clarity and in a nonjudgemental manner that is free from emotional bias. This is typically achieved by engaging in exercises, for example, meditation, breathing and focused attention that settle the mind and provide the conditions under which cross-cultural issues can be explored. The cross-cultural coach can ask a series of incisive questions that direct the client's attention to the thoughts, feelings and sensations in relation to a particular cross-cultural issue, and help the client to explore the issue from different perspectives by identifying automatic biases and responses. Finally, the coach can assist the client in recognising that there is a range of behavioural alternatives, consistent with the client's motivations, for a particular crosscultural interaction, and that this is a conscious choice in the moment of interaction.

Metacognition, exercised through the aforementioned metacognitive strategies, more generally enables an individual to be consciously aware of the cultural preferences of others before and during interactions, to question cultural assumptions and to adjust mental models during and after interactions. As a metacognitive process, it is used to acquire and understand knowledge, suspend judgement and maintain real-time consciousness of how culture influences the mental process and behaviour of oneself and others in intercultural situations (Flavell, 1979). Metacognition therefore allows individuals 'to develop heuristics for social interaction across cultural contexts' (Van Dyne et al., 2012, p. 299). Metacognition has two immediate applications for cross-cultural coaching. 
Firstly, reflective practice is used in cross-cultural coaching to create insight through awareness, perspective-taking and mindfulness, and is widely used in coaching. Various forms of cultural profiling are often used in reflective practice exercises. Although cultural profiling is useful, it does not address the question of how knowledge and experience of culture(s) is transformed into culturally appropriate behaviour. There is limited evidence in the literature on cross-cultural coaching to suggest that the insights of metacognitive CQ are meaningfully integrated into reflective practice in cross-cultural coaching. More recent literature on cross-cultural coaching continues to draw heavily on the foundational work of Hofstede and subsequently the Global Leadership and Organizational Behaviour Effectiveness (GLOBE) project (House et al., 2004). These studies offer empirical data that provide useful tools for the definition and categorisation of cultural artefacts. A number of authors (Abbott et al., 2013; DeLay \& Dalton, 2006; Jenkins, 2006; Rosinski \& Abbott, 2006) have acknowledged, discussed and made progress with the integration of the insight of metacognition in cross-cultural coaching. Booysen (2015) has connected CQ and the concept of Global Mindsets (Javidan, Teagarden, \& Bowen, 2010) in cross-cultural coaching, and provides a powerful cross-cultural coaching framework that inculcates CQ in the development of cultural agility (2015, p. 278). Abbott et al. (2013, p, 492) point out that CQ and Global Mindsets are crucially 'useful heuristics to promote more rigorous and high impact coaching engagements in intercultural contexts'.

Secondly, metacognition is useful as it provides the coach and client with a set of methods, being the metacognitive strategies, to explore cross-cultural challenges in a manner that takes cognisance of the implicit perceptions and bias built into the everyday assessment and understanding of culture, in situations characterised by cultural difference and complexity. Booysen (2015) provides a coaching-specific set of practical steps for building CQ. Journaling and reflective and reflexive practices are identified as effective tools for developing metacognition. A CQ-based approach recognises the significance and role of metacognition, can assist cross-cultural coaching in a manner that supports the ability to improve awareness by enabling experience to be grasped and transformed into learning (Kolb, 1984), and ultimately translated into an adaption of one's behaviour. Booysen (2015) refers to continuous questioning of cultural assumptions when working on developing metacognition. A CQ-based approach can engender the mental agility to be able to, in the moment, carry one's experience to that situation and stand back, assessing appropriately what the situation demands, thereby enabling greater agency when faced with a culturally diverse situation.

\section{Significance of cultural intelligence to experiential learning theory}

The second area of research, relevant to the conceptual case for a CQ-based approach to cross-cultural coaching, is the relationship between CQ and ELT. Two linkages between CQ and ELT are of interest: firstly, the interaction between the components of CQ and the stages of Kolb's experiential learning cycle, and secondly, the moderating impact that the theory of Kolb's learning styles has on improving CQ. These connections have far-reaching implications for cross-cultural coaching.

The literature on CQ increasingly recognises the importance of experiential approaches to development and learning in cross-cultural contexts. Research has focused primarily on cross-cultural questions in the context of leadership, some of which are the impact of international experience on business outcomes (Carpenter, Sanders, \& Gregersen, 2001; Daily, Certo, \& Dalton, 2000; Sambharya, 1996), individual professional development (Gregersen, Morrison, \& Black, 1998; Jokinen, 2005; McCall \& Hollenbeck, 2002) and the importance of cultural proficiency of those in leadership positions (Deal, Leslie, Dalton, \& Ernst, 2003; Javidan et al., 2010). Until Ng, Van Dyne and Ang (2009) proposed the link between CQ and Kolb's ELT, the literature said very little about how to increase CQ. This link was a significant development as it brought together two distinct fields of study in a manner that has advanced the theory and practice of CQ with potentially far-reaching implications for crosscultural coaching. Building on this, Lorenz, Ramsey and Richey (2018) connect ELT to metacognitive and cognitive CQ to explain opportunity recognition in international contexts.

Kolb's ELT is a four-stage cycle, which comprises concrete experience, reflective observation, abstract conceptualisation and active experimentation (Kolb, 1984). Figure 1 depicts Kolb's stages of the experiential learning cycle, discussed below, and the ELS, discussed later in this article. Concrete experience (feeling) is concerned with an immediate, tangible experience. Reflective observation (perceiving) is an internal processing of experience. Abstract conceptualisation (thinking) is concerned with the abstraction, aggregation, classification and symbolic representation of experience, and active experimentation (doing) comprises the testing of new behaviour through the manipulation of the external world.

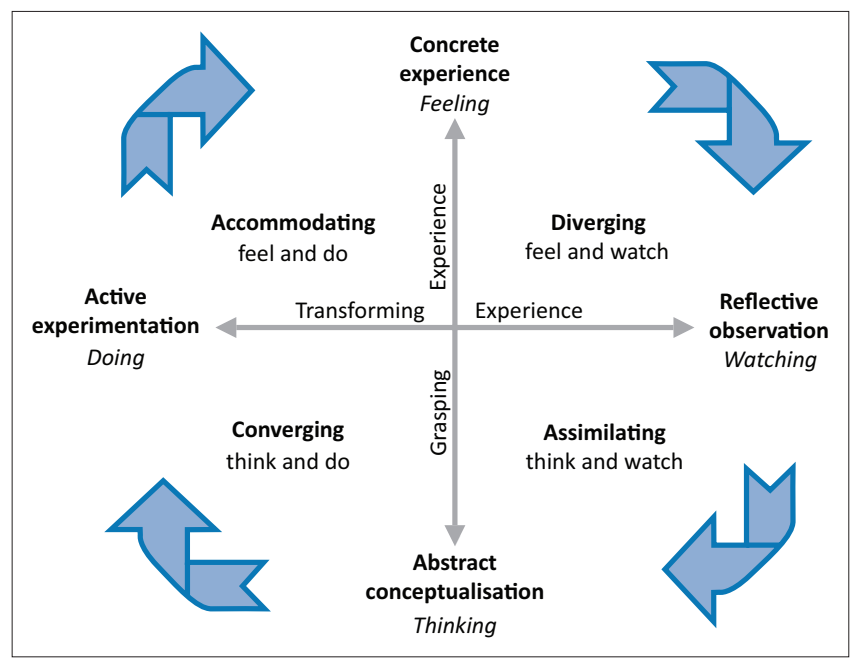

Source: Adapted from Kolb, D. A. (1984). Experiential learning. Englewood Cliffs, NJ: Prentice Hall

FIGURE 1: Kolb's learning cycle and experiential learning styles. 
Kolb's learning cycle assists with understanding experience in order to transform it into useable knowledge (Kolb, 1984). Experiential learning and unlearning take place as a result of grasping experience (concrete experience and abstract conceptualisation) and transforming experience (reflective observation and active experimentation).

Kolb (1984) identified four ELS, namely divergent, convergent, assimilative and accommodative. Each learning style, a function of individual circumstances and preference, occupies a quadrant on Kolb's learning cycle through the combination of adjacent learning stages (see Figure 1). The divergent learning style combines concrete experience and reflective observation and is associated with persons that embrace multiple perspectives and are imaginative in problem resolution. The assimilative learning style combines reflective observation and abstract conceptualisation and is associated with persons that dwell in the world of abstractions and effortlessly master conceptually complex ideas in a logical and concise manner. The convergent learning style combines abstract conceptualisation and active experimentation and is associated with persons with a thought-derived practical approach to problem resolution. Lastly, the accommodative learning style combines active experimentation and concrete experience and is associated with persons who are practical and intuitive (i.e. through the experience of trial and error) in their approach to problem solving.

Experiential learning theory is widely used in coaching. The integration of CQ and ELT (and by implication ELS) may provide a useful process for addressing the complexities associated with cross-cultural issues. $\mathrm{Ng}$ et al. (2009, p. 520) propose a framework that identifies and integrates different stages of ELT with CQ, and suggest that positive outcomes from cross-cultural learning experiences can potentially lead to higher levels of CQ. Higher levels of CQ enhance the likelihood of active involvement in all four stages of the ELT cycle, resulting in better integration of the ELT processes of grasping experience and transforming experience ( $\mathrm{Ng}$ et al., 2009, p. 523). This is an ongoing and mutually reinforcing process, as higher levels of CQ facilitate progression through the experiential learning cycle. Iterations in the completed experiential learning cycle continue to develop CQ. The aforementioned research suggests that improving CQ through the cross-cultural coaching process can potentially unlock the client's ability to access Kolb's full experiential learning cycle. While an empirical foundation continues to be established for the linkages between CQ and ELT, Ng et al. (2009) propose four key relationships: Firstly, an individual with higher levels of motivational and/or behavioural CQ will in all likelihood seek concrete experience and develop higher levels of self-efficacy in cross-cultural settings. Motivational and behavioural CQ are the operative components of $C Q$, which make an individual open to new cross-cultural experiences, take greater interest and initiative, and learn from doing and feeling rather than thinking. Research by Bandura (1997) suggests that self-confidence is necessary for persistence and task completion, which is connected to motivational CQ. In a cross-cultural setting or an unfamiliar environment, higher levels of motivational CQ predispose the individual to seeking out, rather than avoiding, concrete experience. Higher levels of behavioural CQ arguably lead to culturally more appropriate and flexible behaviour as the individual values concrete experience as a form of learning and engagement with others and the environment, and seeks out such interactions with greater frequency.

Secondly, people with strong metacognitive and cognitive CQ abilities are more likely to engage in reflective observation as a form of learning and develop ethnorelative attitudes towards other cultures (Ang, Van Dyne, \& Rockstuhl, 2015; $\mathrm{Ng}$ et al., 2009). Lorenz et al. demonstrate the positive impact of metacognition and cognition on innovativeness, the product of learning and the application of experience, in an international context (2018). Mental representations of culture reside within the cognitive faculties of CQ. Metacognition bolsters the ability to derive rich and accurate mental schema of culture and cultural interactions through the ongoing monitoring and adjustment of cognition and the structure of thought (Flavell, 1979). Persons with highly developed metacognitive and cognitive abilities tend to make better cultural assessments and are better equipped to engage in the process of reflective observation through a heightened understanding of the similarities and differences in culture (Ang et al., 2007).

Thirdly, heightened levels of metacognitive and cognitive CQ will in all likelihood result in improved pattern detection and abstract conceptualisation. Abstract conceptualisation is concerned with deriving general propositions, theories, models, patterns and schema about interactions and the environment. Metacognition supports the process of abstract conceptualisation by providing the mental wherewithal to reflect on the structure and content of thought, and monitor and adjust ongoing basic assumptions, values, feelings and representations. Persons with accurate mental models and better organised knowledge structures about their environments are thought to be better equipped, in part, for effective cross-cultural interactions.

Lastly, Ng et al. (2009) suggest that all four components of $C Q$, in varying combinations, are necessary and contribute to an individual implementing and testing conceptualised generalisations through active experimentation, which facilitates the development of the appropriate skills and flexibility for cross-cultural interactions. The process of active experimentation employs the use of all four components of $C Q$. Both metacognitive and cognitive CQ are required to organise and map out action plans. Motivational CQ provides the interest, focus and persistence to engage in new behaviour, and behavioural CQ facilitates meaningful verbal and non-verbal interaction (Ng et al., 2009, p. 517).

\section{Applications for cross-cultural coaching}

Cultural intelligence and Kolb's experiential learning theory

While there has been significant theoretical development in the theory and application of CQ and ELT, this research has 
further identified a number of implications for cross-cultural coaching from the integration of CQ and ELT:

- Cross-cultural coaching can assist clients in accessing and engaging all four experiential learning stages to help them improve CQ (Li et al., 2013; Lorenz et al., 2018) by progressing through the experiential learning cycle in the grasping and transformation of experience into culturally appropriate behaviour.

- The literature points to concrete experience, reflective observation and active experimentation as learning stages that enhance CQ. Coaching can assist particularly with the process of reflective and reflexive practice (Booysen, 2015). Ang et al. (2015) refer to the use of cognitive and metacognitive CQ abilities in reflective observation. Reflexive practice includes the taking of multiple perspectives (Kolb, 1984), suspending judgement (Triandis, 2006), raising awareness (Whitmore, 2009) and cultivating mindfulness (Thomas, 2006) for positive relational outcomes (Kopelman et al., 2011). In addition, the coaching conversation can create the environment in which possible forms of active experimentation can be explored.

- Coaching can assist with abstract conceptualisation, a metacognitive process, by assisting the client with pattern recognition and thinking through the systemic aspects of cross-cultural experience. Learning takes place in the contextual relevance of change situations and not because of positions of externality (Su, 2011). Coaching can provide an environment in which the client can reflect on previous experience; challenge mental schemata, frames and assumptions; and integrate learning.

- Thomas (2006) and Ang and Van Dyne (2008) propose that metacognition links the cognitive and behavioural elements of CQ by facilitating the translation of thought into action owing to ongoing active awareness that leads to situation-appropriate behaviour. When this theory is overlaid with Kolb's ELT theory, it becomes apparent that 'even if people know what they should do and have the necessary motivation, it does not always mean they will enact the behaviours' (Li et al., 2013, p. 34). Coaching, as an ongoing process, can, however, support the transformative learning (Terblanche, Albertyn, \& Van Coller-Peter, 2018) necessary for the enactment of culturally appropriate behaviour. This process assists coachees to move from 'knowing' to 'becoming' through action, learning and experience (Yeo \& Marquardt, 2015, p. 81).

\section{Cultural intelligence and Kolb's experiential learning styles}

In recent years, research has been directed at the development of conceptual models of learning that account for the learning processes underpinning heightened $\mathrm{CQ}$ and improved organisational outcomes that can specify the type of individual best suited to cross-cultural interactions ( $\mathrm{Ng}$ et al., 2009). Yamazaki and Kayes (2004) found that certain ELS were better suited to particular cross-cultural contexts and resulted in more effective learning. In particular, abstract conceptualisation was found to be of lesser importance for cultural adaption than concrete experience, reflective observation or active experimentation. MacNab (2012) demonstrated the positive impact of an ELT-based programme on CQ. Li et al. (2013) investigated the extent to which learning style influences the improvement of CQ in international assignments and found a positive relationship between the length of an international assignment and CQ. This relationship is strengthened for persons with a divergent (not an assimilative, convergent or accommodative) learning style and enhances the likelihood that the individual engaged the four stages of the learning cycle. Lorenz et al. suggest that the components of metacognition and cognition relate to the assimilation and convergent learning styles (2018). The research of Kolb (1984); Kolb and Kolb (2005); $\mathrm{Ng}$ et al. (2009); Li et al. (2013) and Lorenz et al. (2018) have four important implications for cross-cultural coaching:

- Kolb (1984) recognises that a person does not touch all four stages of the experiential learning cycle and that ELT is not a true reflection of how people learn (Kolb, 1984). In the process of learning, a person does not logically and systematically step through the four stages of learning in sequence, but rather jumps around the four stages and does so on the basis of a predisposed learning style. This insight provides a valuable starting point for client and coach, as the client's attention can be directed at potential blind spots and the coach can support the learning process on the basis of the relative strengths and weaknesses of the client's learning style.

- The literature points to a positive relationship between the diverging learning style and cultural adaption (Li et al., 2013). This insight can frame and support the cross-cultural coaching engagement, and direct the focus, if necessary, to developing aspects of concrete experience and reflective observation. The metacognitive strategies of awareness, mindfulness and perspective-taking offer powerful reflective tools that can be integrated with ELT to develop the learning stages of concrete experience and reflective observation.

- Lorenz et al. (2018) suggest that a focus on the development of the metacognitive and cognitive components of CQ (in the coaching engagement) can lead to higher levels of metacognitive and cognitive $C Q$, which are the capabilities underlying the mental flexibility, competence and adaptability required for the successful navigation of crosscultural interactions The development of metacognitive $\mathrm{CQ}$, which is associated with the assimilative learning style, can be supported through different forms of reflective and reflexive practice in the coaching engagement.

- The significance of the development of cognitive CQ, which is associated with the convergent learning style and idea generation, is highlighted by Lorenz et al. (2018). Cognitive CQ can be developed by in-depth learning of other cultural contexts through the experiences of others presently or previously immersed in the cross-cultural context. The coach can support this learning process by providing the impetus for such learning and an environment for reflection that is critical of one's own world view and the framing of other perspectives (Lorenz et al., 2018) 


\section{Conclusion}

The importance of CQ is increasingly recognised in the literature as leaders increasingly need a Global Mindset (Booysen, 2015). There is substantial reference to CQ in adjacent fields of study, but surprisingly limited reference in the literature on cross-cultural coaching to CQ and the benefits to be gained from a CQ-based cross-cultural coaching approach. ${ }^{2}$ A considerable body of research on CQ and related concepts seeks to understand cultural adaption and the transformation of experience into culturally appropriate behaviour. In cross-cultural coaching, however, the question of cultural adaption continues to be addressed through static and dimensionalised cultural profiling methods that focus almost exclusively on the content of culture. Apart from a number of studies cited in this article, there is limited evidence of a body of research comprising empirical evidence and the theoretical development thereof in cross-cultural coaching relating to methods that seek to transform cultural experience and knowledge into culturally appropriate behaviour. This article has made a case for the use of a CQ-based approach in cross-cultural coaching by integrating metacognition and Kolb's ELT. Insights in this article could provide cross-cultural coaches with a more integrated mechanism and tools to facilitate the development of CQ in a multicultural setting.

From a theoretical perspective, the concept of CQ reflects the nebulous, complex and interdisciplinary nature of this field of study. Empirical research on CQ is ongoing. The fields of CQ and cross-cultural coaching, amongst others, stand to benefit from further empirical research on intelligence and metacognition in particular. Cross-cultural coaching can benefit from further conceptual clarification and definition of the metacognitive strategies of awareness, perspective-taking and mindfulness as the terms are used widely, variously and interchangeably. Further empirical research is required to investigate and validate the linkages between CQ and ELT. This research may provide a basis upon which quantitative and qualitative assessments can be made with respect to the likelihood of the attainment of specific cross-cultural outcomes and the suitability of certain personality types to cross-cultural assignments. These are exciting and important developments as the applications extend beyond crosscultural coaching to the direct and, arguably, measurable attainment of individual and organisational outcomes in cross-cultural settings.

From a practitioner's perspective, a CQ-based approach is proposed for cross-cultural coaching. It is argued, firstly that the metacognitive strategies of awareness, perspective-taking and mindfulness can make a meaningful contribution to the cross-cultural coaching process. These metacognitive strategies are already widely used by coaches. However, recognising the importance of metacognition for the transformation of experience into culturally appropriate behaviour significantly raises the usefulness of metacognitive

2.A Google Scholar keyword search for cultural intelligence and cross-cultural coaching
yielded 11 results. Similarly, a keyword search for cultural intelligence and psychology yielded 11 results. Similarly, a keyw
returned in excess of 8000 results. strategies in cross-cultural coaching. Secondly, the adoption of a CQ-based approach to cross-cultural coaching can potentially lead to unlocking the full experiential learning cycle in crosscultural coaching. Experiential learning theory is widely used in coaching. Understanding the linkages between CQ and experiential learning stages has an impact on how coach and client approach the cross-cultural coaching engagement, and can make a meaningful difference to the usefulness of cross-cultural coaching. Similarly, recognising the inherent strengths and limitations of a client's ELS also has an impact on the client and opens up the cross-cultural coaching conversation to assumptions and questions of suitability.

To conclude, adopting a CQ-based approach could enrich and enhance the cross-cultural coaching engagement as it layers insight from CQ on well-established coaching methods in addressing cross-cultural issues.

\section{Acknowledgements Competing interests}

The authors declare that they have no financial or personal relationships that may have inappropriately influenced them in writing this article.

\section{Authors' contributions}

C.A.v.d.H. was involved with the conceptual motivation for a CQ-based framework for cross-cultural coaching, while R.M.A. supervised this project.

\section{References}

Abbott, G., Gilbert, K., \& Rosinski, P. (2013). Cross-cultural working in coaching and mentoring. In J. Passmore, D. B. Peterson, \& T. Freire (Eds.), The Wiley-Blackwell handbook of the psychology of coaching and mentoring (pp. 483-500). Chichester, UK: Wiley.

Ang, S., \& Van Dyne, L. (2008). Conceptualization of cultural intelligence: Definition, distinctiveness and nomological network. In S. Ang \& L. van Dyne (Eds.), Handbook of cultural intelligence: Theory, measurement, and applications (pp. 3-15). New York: Sharpe.

Ang, S., Van Dyne, L., Koh, C., Ng, K.Y., Templer, K.J., Tay, C., \& Chandrasekar, N.A. (2007). Cultural Intelligence: Its measurement and effects on cultural judgement and decision making, cultural adaption and task performance. Management and Organization Review, 3(3), 335-371. https://doi.org/10.1111/j.1740-8784.2007.00082.x

Ang, S., Van Dyne, L., \& Rockstuhl, T. (2015). Cultural intelligence: Origins, conceptualization, evolution and methodological diversity. In Gelfand, M.J.,
Chiu, C. \& Hong, Y. (Eds.), Handbook of advances in culture and psychology (Vol. 5, pp. 1-45).

Bandura, A. (1997). Self-efficacy: The exercise of self-control. New York: Freeman.

Barnett-Page, E., \& Thomas, J. (2009). Methods for the synthesis of qualitative research: A critical review. BMC Medical Research Methodology, 9(1), 1. https:// doi.org/10.1186/1471-2288-9-59

Booysen, L.A.E. (2015). Cross-cultural coaching. In D. Riddle, E. Hoole, \& E. Gullette (Eds.), Center for creative leadership handbook of coaching in organizations (pp. 241-288). San Francisco, CA: Wiley, Jossey-Bass.

Brown, K.W., \& Ryan, R.M. (2003). The benefits of being present: Mindfulness and its role in psychological well-being. Journal of Personality and Social Psychology 84(4), 822. https://doi.org/10.1037/0022-3514.84.4.822

Carpenter, M.A., Sanders, W.G., \& Gregersen, H.B. (2001). Bundling human capital with organizational context: The impact of international assignment experience on multinational firm performance and CEO pay. Academy of Management Journal, 44(3), 493-511. https://doi.org/10.2307/3069366

Chua, R.Y.J., Morris, M.W., \& Mor, S. (2012). Collaborating across cultures: Cultural metacognition and affect-based trust in creative collaboration. Organizational Behavior and Human Decision Processes, 118, 116-131. https://doi.org/10.1016/j. obhdp.2012.03.009

Daily, C.M., Certo, S.T., \& Dalton, D.R. (2000). Research notes and communications: International experience in the executive suite: The path to prosperity. Strategic Management Journal, 21, 515-523. https://doi.org/10.1002/(SICI)1097-0266 (200004)21:4\%3C515::AID-SMJ92\%3E3.0.CO;2-1 
Dane, E. (2011). Paying attention to mindfulness and its effects on task performance in the workplace. Journal of Management, 37(4), 997-1018. https://doi.org/ in the workplace. Journal of

Deal, J.J., Leslie, J., Dalton, M., \& Ernst, C. (2003). Cultural adaptability and leading across cultures. Advances in Global Leadership, 3, 149-166. https://doi.org/ 10.1016/S1535-1203(02)03008-3

DeLay, L., \& Dalton, M. (2006). Coaching across cultures. In S. Ting \& P. Scisco (Eds.), CCL handbook of coaching: A guide for the leader coach (pp. 122-148). San Francisco, CA: Jossey-Bass.

Deng, L., \& Gibson, P. (2008). A qualitative evaluation on the role of cultura intelligence in cross-cultural leadership effectiveness. International Journal of Leadership Studies, 3(2), 181-197.

Dickson, M.W., Den Hartog, D.N., \& Mitchelson, J.K. (2003). Research on leadership in a cross-cultural context: Making progress and raising new questions. The Leadership Quarterly, 14, 729-768. https://doi.org/10.1016/j.leaqua.2003.09.002

Dixon-Woods, M., Cravers, D., Agarwal, S., Annandale, E., Arthur, A., Harvey, J., ... Sutton, A. J. (2006). Conducting a critical interpretive synthesis of the literature on access to healthcare by vulnerable groups. BMC Medical Research Methodology, 6, 35. https://doi.org/10.1186/1471-2288-6-35

Earley, P.C., \& Ang, S. (2003). Cultural intelligence: Individual interactions across cultures. Stanford: Stanford University Press.

Earley, P.C., \& Gibson, C.B. (2002). Multinational work teams: A new perspective. New York: Routledge.

Earley, P.C., \& Mosakowski, E. (2004). Cultural intelligence. Harvard Business Review, October, pp. 139-146.

Earley, P.C., Murnieks, C., \& Mosakowski, E. (2007). Cultural intelligence and the global mindset. Advances in International Management, 19(1), 75-103. https://doi. org/10.1016/S1571-5027(07)19004-5

Elenkov, D.S., \& Manev, I.M. (2009). Senior expatriate leadership's effects on innovation and the role of cultural intelligence. Journal of World Business, 44(4), 357-369. https://doi.org/10.1016/j.jwb.2008.11.001

Endsley, M.R. (1995). Toward a theory of situation awareness in dynamic systems. Human Factors: The Journal of the Human Factors and Ergonomics Society, 37(1), 32-64. https://doi.org/10.1518/001872095779049543

Flavell, J. H. (1979). Metacognition and cognitive monitoring: A new area of cognitivedevelopmental inquiry. American Psychologist, 34(10), 906. https://doi.org/ 10.1037/0003-066X.34.10.906

Galinsky, A.D. (2002). Creating and reducing intergroup conflict: The role of perspective-taking in affecting out-group evaluations. In H. Sondak (Ed.), Research
on managing groups and teams, Vol. 4. Toward phenomenology of groups and on managing groups and teams, Vol. 4. Toward phenomenology of groups and
group membership (pp. 85-113). New York, NY, US: Elsevier Science. http://dx.doi. org/10.1016/S1534-0856(02)04005-7

Galinsky, A.D., \& Moskowitz, G.B. (2000). Perspective-taking: Decreasing stereotype expression, stereotype accessibility, and in-group favoritism. Journal of Personality and Social Psychology, 78(4), 708. https://doi.org/10.1037/0022-3514.78.4.708

Geertz, C. (1973). The interpretation of cultures. New York: Basic Books.

Hampden-Turner, C., \& Trompenaars, F. (1997). Riding the waves of culture. London: Nicholas Brealey.

Gregersen, H.B., Morrison, A.J., \& Black, J.S. (1998). Developing leaders for the global frontier. MIT Sloan Management Review, 40(1), 21

Hofstede, G. (1981). Culture and organizations. International Studies of Management and Organization, 10(4), 15-41. https://doi.org/10.1080/00208825.1980.1165 6300

House, R.J., Hanges, P.J., Javidan, M., Dorfman, P.W., \& Gupta, V. (2004). Culture, leadership, and organizations: The GLOBE study of 62 societies. Thousand Oaks, CA: Sage.

Javidan, M., \& House, R.J. (2002). Leadership and cultures around the world: Findings from GLOBE: An introduction to the special issue. Journal of World Business, 37(1), 1-2. https://doi.org/10.1016/S1090-9516(01)00068-2

Javidan, M., Teagarden, M., \& Bowen, D. (2010). Managing yourself making it overseas. Harvard Business Review, 88(4), 109-113.

Jenkins, J. (2006). Coaching meets the cross-cultural challenge. Leadership in Action, 26(5), 23-24. https://doi.org/10.1002/lia.1182

Johnson, J.P., Lenartowicz, T., \& Apud, S. (2006). Cross-cultural competence in international business: Toward a definition and a model. Journal of Internationa Business Studies, 37(4), 525-543. https://doi.org/10.1057/palgrave.jibs.8400205

Jokinen, T. (2005). Global leadership competencies: A review and discussion. Journal of European Industrial Training, 29(3), 199-216. https://doi.org/10.1108/0309 0590510591085

Kabat-Zinn, J. (2003). Mindfulness-based interventions in context: Past, present and future. Clinical Psychology: Science and Practice, 10(2), 144-156. https://doi. org/10.1093/clipsy.bpg016

Klafehn, J., Banerjee, P.M., \& Chiu, C.Y. (2008). Navigating cultures. In Handbook of cultural intelligence: Theory, measurement, and applications (p. 318). New York: Sharpe.

Kolb, A.Y., \& Kolb, D.A. (2005). The Kolb learning style inventory - Version 3.1. Technical specifications. Boston, MA: Hay Resources Direct.

Kolb, D. A. (1984). Experiential learning. Englewood Cliffs, NJ: Prentice Hall.

Kopelman, S., Avi-Yonah, O. \& Varghese, A. The Mindful Negotiator: Strategic Emotion Management and Wellbeing (June 17, 2011). In IACM 2011 Istanbul Conference Paper. http://dx.doi.org/10.2139/ssrn.1866504
Langer, E.J. (1989). Mindfulness. Reading, MA: Addison-Wesley/Addison Wesley Longman.

Lee, S., Adair, W.L., \& Seo, S.J. (2013). Cultural perspective taking in cross-cultural negotiation. Group Decision Negotiation, 22, 389-405. https://doi.org/10.1007/ s10726-011-9272-4

Li, M., \& Mobley, W.H. (2010, August). The role of experiential learning in the development of cultural intelligence. Paper delivered at the Annual Meeting of the Academy of Management, Montreal, Canada.

Li, M., Mobley, W.H., \& Kelly, A. (2013). When do global leaders learn best to develop $\mathrm{Cl}$ ? An investigation of the moderating role of experiential learning style. Academy of Management Learning \& Education, 12(1), 32-50. https://doi. org/10.5465/amle.2011.0014

Livermore, D.A. (2009). Cultural intelligence: Improving your CQ to engage our multicultural world. Grand Rapids, MI: Baker Publishing Group.

Logan, G.D. (1989). Automaticity and cognitive control. In J.S. Uleman \& J.A. Bargh (Eds.), Unintended thought (pp. 52-74). New York: Guilford.

Lorenz, P.L., Ramsey, J.R., \& Richey, R.G. (2018). Expatriates' international opportunity recognition and innovativeness: The role of metacognition and cognitive cultural intelligence. Journal of World Business, 53, 222-236. https://doi.org/10.1016/j. jwb.2017.11.004

MacNab, B.R. (2012). An experiential approach to cultural intelligence education. Journal of Management Education, 36(1), 66-94. https://doi.org/10.1177/10525 62911412587

McCall, M.W., \& Hollenbeck, G.P. (2002). Developing global executives: The lessons of international experience. Boston, MA: Harvard Business School Press.

Meyer, E. (2014). The culture map: Breaking through the invisible boundaries of global business. New York: Public Affairs.

Mor, S., Morris, M., \& Joh, J. (2013). Identifying and training adaptive cross-cultural management skills: The crucial role of metacognition. Academy of Management Learning \& Education, 12(3), 453-475. https://doi.org/10.5465/amle.2012.0202

Ng, K.Y., Van Dyne, L., \& Ang, S. (2009). From experience to experiential learning: Cultural intelligence as a learning capability for global leader development. Academy of Management Learning \& Education, 8(4), 511-526. https://doi. Academy of Management Learning
org/10.5465/AMLE.2009.47785470

Ng, K.Y., Van Dyne, L., \& Ang, S. (2012). Cultural intelligence: A review, reflections, and recommendations for future research. In A.M. Ryn, F.T.L. Leong, \& F.L. Oswald (Eds.), Conducting multinational research: Applying organizational psychology in the workplace (pp. 29-58). Washington, DC: American Psychological Association.

Oolders, T., Chernyshenko, O., \& Stark, S. (2008). Cultural intelligence as mediators of relationships between openness to experience and adaptive performance. In $\mathrm{S}$. Ang \& L. van Dyne (Eds.), Handbook of cultural intelligence: Theory, measurement, and applications (pp. 145-158). New York: Sharpe.

Plaister-Ten, J. (2009). Towards greater cultural understanding in coaching. International Journal of Evidence Based Coaching and Mentoring Special Issue (3), 64-81.

Plaister-Ten, J. (2013). Raising culturally-derived awareness and building culturallyappropriate responsibility: The development of the cross-cultural kaleidoscope. International Journal of Evidence Based Coaching and Mentoring, 11, 53-69.

Rosinski, P. (2003). Coaching across cultures. International Journal of Coaching in Organizations, 1(4), 4-16.

Rosinski, P., \& Abbott, G.N. (2006). Coaching from a cultural perspective. In D.R. Stober \& A.M. Grant (Eds.), Evidence based coaching handbook: Putting best practices to work for your clients (pp. 255-275). Hoboken, NJ: Wiley.

Rowland, D. (2016). Leading across cultures requires flexibility and curiosity. Harvard Business Review, May 30. https://hbr.org/2016/05/leading-across-culturesrequires-flexibility-and-curiosity

Sambharya, R.B. (1996). Research notes and communications: Foreign experience of top management teams and international diversification strategies of US multinational corporations. Strategic Management Journal, 17, 739-746. https:// doi.org/10.1002/(SICI)1097-0266(199611)17:9\%3C739::AID-SMJ846\%3E3.0. CO;2-K

Schoenfeld, A.H. (1987). What's all the fuss about metacognition? In A.H. Schoenfeld (Ed.), Cognitive science and mathematics education (pp. 1-31). Hillsdale, NJ: Lawrence Erlbaum.

Schreiber, R., Crooks, D., \& Stern, P.N. (1997). Qualitative meta-analysis. In J.M. Morse (Ed.), Completing a qualitative project: Details and dialogue (pp. $311-326$ ). London: Sage.

Sheldon, K.M. (1996). The social awareness inventory: Development and applications Personality and Social Psychology Bulletin, 22(6), 620-634. https://doi.org/ 10.1177/0146167296226007

Silver, E.A. (1987). Foundations of cognitive theory and research for mathematics problem- solving. In A.H. Schoenfeld (Ed.), Cognitive science and mathematics education (pp. 33-60). Hillsdale, NJ: Lawrence Erlbaum.

St Claire-Ostwald, B. (2007). Carrying cultural baggage: The contribution of sociocultural anthropology to cross-cultural coaching. International Journal of Evidence Based Coaching and Mentoring, 25(2), 45-52.

Sternberg, R.J. (2004). Culture and intelligence. American Psychologist, 59(5), 325. https://doi.org/10.1037/0003-066X.59.5.325

Su, Y. (2011). Lifelong learning at being: The Heideggerian perspective. Adult Education Quarterly, 61(1), 57-72. https://doi.org/10.1177/0741713610380442

Tan, J.S., \& Chua, R.Y. (2003). Training and developing cultural intelligence. In P.C. Earley \& S. Ang (Ed.), Cultural intelligence: Individual interactions across cultures. (pp. 258 - 303). Stanford: Stanford University Press. 
Terblanche, N., Albertyn, R.M., \& Van Coller-Peter, S. (2018). Using transformative transition coaching to support leaders during career transitions. African Journal of Business Ethics.

Thomas, D.C. (2006). Domain and development of cultural intelligence: The importance of mindfulness. Group \& Organization Management, 31(1), 78-99. https://doi.org/10.1177/1059601105275266

Thomas, D.C., \& Inkson, K. (2003). Cultural intelligence: People skills for global business. San Francisco, CA: Berrett-Koehler Publishers.

Triandis, H.C. (1995). Individualism and collectivism. Boulder, CO: Westview.

Triandis, H.C. (2006). Cultural intelligence in organizations. Group \& Organization Management, 31(1), 20-26. https://doi.org/10.1177/1059601105275253

Van Dyne, L., Ang, S., \& Livermore, D. (2009). Cultural intelligence: A pathway for leading in a rapidly globalizing world. In K. M Hannum, B McFeeters \& L. Booysen (Eds.), Leadership across differences: Cases and perspectives. (pp.131 - 138).San Francisco, CA: Pfeiffer.
Van Dyne, L., Ang, S., Ng, K.Y., Rockstuhl, T., Tan, M.L., \& Koh, C. (2012). Sub-dimensions of the four factor model of cultural intelligence: Expanding the conceptualisation and measurement of Cl. Social and Personality Psychology Compass, 6(4), 295-313. https://doi.org/10.1111/j.1751-9004.2012.00429.x

Walsh, R. (1980). The consciousness disciplines and the behavioral sciences: Questions of comparison and assessment. American Journal of Psychiatry, 137(6), 663-673. https://doi.org/10.1176/ajp.137.6.663

Whitmore, J. (2009). Coaching for performance (4th ed.). Boston, MA: Nicholas Brealey Publishing.

Yamazaki, Y., \& Kayes, D.C. (2004). An experiential approach to cross-cultural learning A review and integration of competencies for successful expatriate adaptation. Academy of Management Learning \& Education, 3(4), 362-379. https://doi.org/ 10.5465/AMLE.2004.15112543

Yeo, R.K., \& Marquardt, M.J. (2015). (Re) Interpreting action, learning and experience: Integrating action learning and experiential learning for HRD. Human Resource Development Quarterly, 26(1), 81-107. https://doi.org/10.1002/hrdq.21199 